\title{
Advanced substrate holder and multi-axis manipulation tool for ultramicrotomy
}

\author{
Waldemar Spomer ${ }^{1,2}$, Andreas Hofmann ${ }^{1,2}$, Irene Wacker ${ }^{2,3}$, Len Ness ${ }^{4}$, Pat Brey ${ }^{4}$, Rasmus R. \\ Schröder ${ }^{2,5}$ and Ulrich Gengenbach ${ }^{1,2}$ \\ ${ }^{1}$ Institute for Applied Computer Science, Karlsruhe Institute of Technology (KIT), Karlsruhe, Germany \\ ${ }^{2}$ HEiKA, Heidelberg Karlsruhe Research Partnership, Heidelberg, Karlsruhe, Germany \\ ${ }^{3}$ Cryo EM, Centre for Advanced Materials, Universität Heidelberg, Heidelberg, Germany \\ ${ }^{4}$ RMC Boeckeler, Tucson, Arizona, USA \\ ${ }^{5}$ Cryo EM, CellNetworks, BioQuant, Universitätsklinikum Heidelberg, Heidelberg, Germany
}

Array tomography (AT) allows reconstructing ultrastructure of large volumes at nano-scale resolution. This nondestructive method preserves sectioned samples and thus enables correlative imaging: Different imaging techniques, such as light microscopy (LM) and electron microscopy (EM), are applied on the same sample [1].

Samples are generated by cutting ultrathin sections and collecting them as ribbons swimming on the surface of a water filled knife trough. The substrate needs to be conductive (for EM) and transparent (for LM) such as indium tin oxide (ITO) coated glass coverslips. While sectioning is done by the ultramicrotome the collecting step is still a tedious, manual handling process and requires a human operator. Typically the substrate is held in one hand while the other manipulates the ribbon. Holding the substrate by hand may be affected by tremor - a major obstacle for perfectly aligned ribbons. Furthermore it is nearly impossible to deposit more than one ribbon on the substrate. There are several concepts to support the operator in this task. Beginning from the 1960s [2] until now [3] devices holding the substrate were presented from time to time. However they do not offer the required degrees of freedom to fit all desired knife orientations. The block-face of the sample mounted in the sample holder is very rarely in parallel with the knife edge. That is a reason why nowadays microtomes offer a yaw axis for knife rotation to produce usable sections right from the beginning of the sectioning process. While the knife is rotated a substrate held by a fixed holder remains in its orientation with respect to the microtome. Hence substrates using the full width of the knife trough may collide with the side of the trough if the knife is being realigned. Thus an adaptation for rotated knifes is a desirable feature of a substrate holder.

Another important aspect is the lift-up movement of the substrate from the trough once the sections have been pinned to its surface. Depending on the type of sections and the substrate surface properties a lift-up trajectory involving several axes simultaneously reduces ribbon damage. The substrate holders proposed so far fall short on these two aspects.

To increase reliability and flexibility in section collection we developed a novel, multi-axis device, which supports the operator in transferring the sections onto a solid substrate such as ITO glass or silicon wafer respectively. With its seven degrees of freedom (Fig. 1A) it enables positioning the substrate in different orientations (lower axes) as well as different lift-up movements (upper axes, Fig. 1B). Moreover, lift-up involving two axes at the same time enables smooth movements with minimal water turbulence and thus avoids ribbon drift and ribbon breaking. The substrate holder provides two axes (x/y) for coarse adjustment and a rotary stage for the rotation about the z-axis to fit rotated knifes. For finer positioning and height adjustment there are micro-positioning stages. With the device presented here thousands of ribbons have been collected even on challenging substrates with rough surface such as ITO-coated glass (Fig. 1C, cf. Wacker et al., this conference). 
References:

[1] KD Micheva, DJ Smith, Neuron 55 (2007), p. 25-36.

[2] O Behnke, J Rostgaard, Biotechnic \& Histochemistry 39 (1964), p. 205-208

[3] H Horstmann et al., PLoS ONE 7 (2012), p. e35172

[4] The authors acknowledge C Bartels, L Veith and R Scharnowell for technical support, as well as the Heidelberg Karlsruhe Research Partnership (HEiKA) for initial funding.

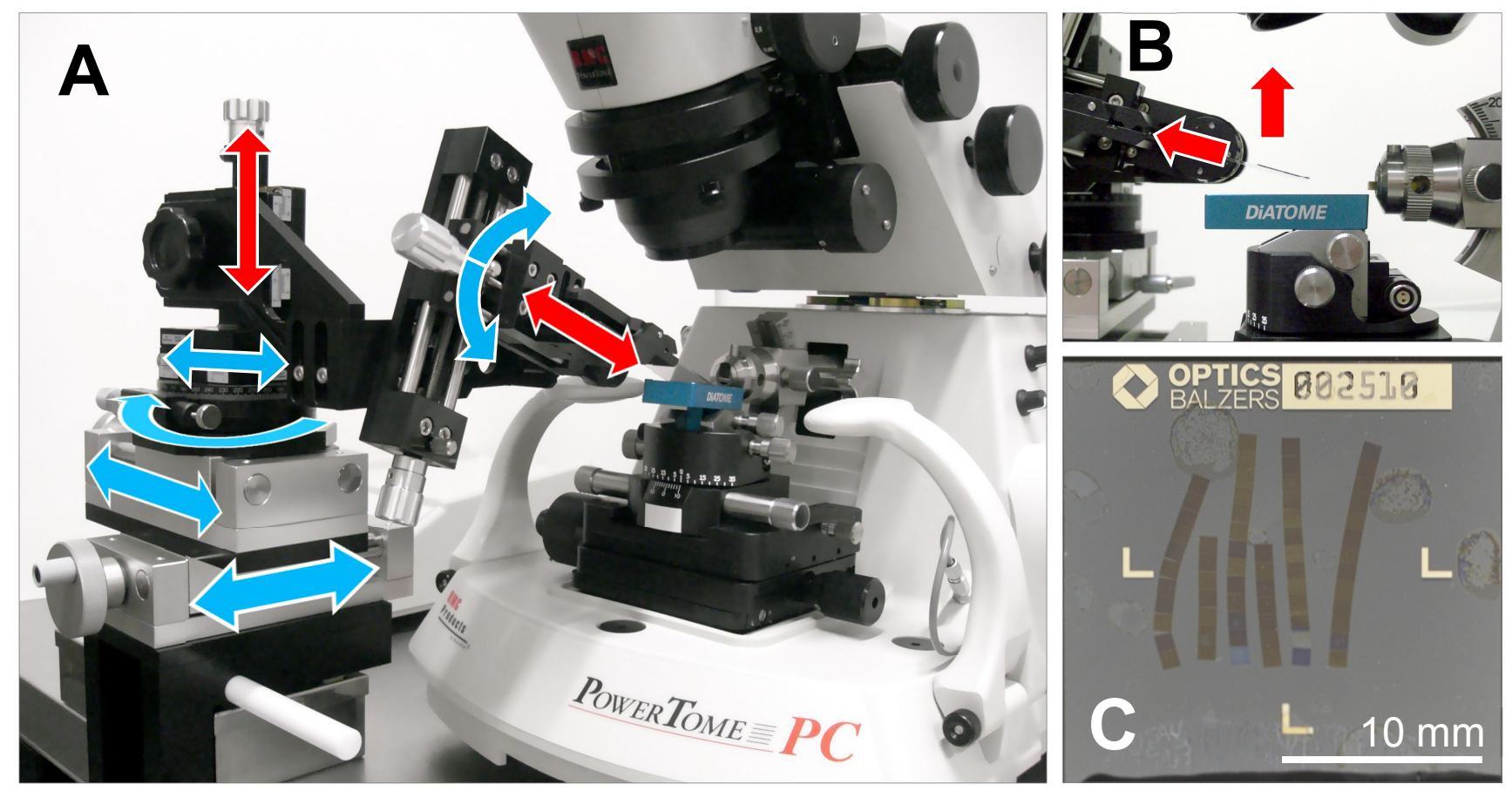

Figure 1: Substrate holder mounted in front of an ultramicrotome with seven adjustable axes for positioning (blue arrows) and lift up (red arrows) (A); Side view: Variable lift-up movements (B); Six ribbons deposited on an ITO-coated glass coverslip using the device presented here (cf. Wacker et al., this conference) $(\mathrm{C})$ 